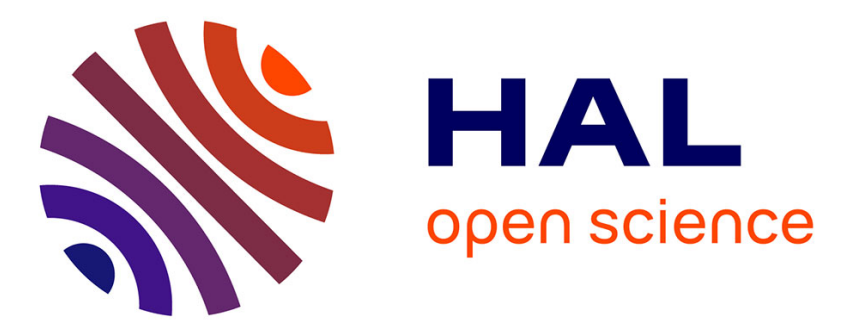

\title{
On choosing a proper basis for determining structures of quasicrystals
}

Frédéric Lançon, Luc Billard, Sergei Burkov, Marc de Boissieu

\section{To cite this version:}

Frédéric Lançon, Luc Billard, Sergei Burkov, Marc de Boissieu. On choosing a proper basis for determining structures of quasicrystals. Journal de Physique I, 1994, 4 (2), pp.283-301. 10.1051/jp1:1994138 . jpa-00246905

\section{HAL Id: jpa-00246905 https://hal.science/jpa-00246905}

Submitted on 1 Jan 1994

HAL is a multi-disciplinary open access archive for the deposit and dissemination of scientific research documents, whether they are published or not. The documents may come from teaching and research institutions in France or abroad, or from public or private research centers.
L'archive ouverte pluridisciplinaire HAL, est destinée au dépôt et à la diffusion de documents scientifiques de niveau recherche, publiés ou non, émanant des établissements d'enseignement et de recherche français ou étrangers, des laboratoires publics ou privés. 
Classification

Physics Abstracts

$61.00-61.55$

\title{
On choosing a proper basis for determining structures of quasicrystals
}

\author{
Frédéric Lançon $\left({ }^{1}\right)$, Luc Billard $\left({ }^{1}\right)$, Sergei Burkov $\left({ }^{1, *}\right)$ and Marc de Boissieu $\left({ }^{2}\right)$ \\ $\left({ }^{1}\right)$ Département de Recherche Fondamentale sur la Matière Condensée, CEA, SP2M/MP, 38054 \\ Grenoble Cedex 9, France \\ $\left({ }^{2}\right)$ Laboratoire de Thermodynamique et de Physico-Chimie Métallurgique, INPG/ENSEEG, \\ B.P. 75, 38402 Saint Martin d'Hères Cedex, France
}

(Received 15 April 1993, revised 22 September 1993, accepted 2 November 1993)

\begin{abstract}
Résumé. - Une erreur d'indexation du diagramme de diffraction d'un quasi-cristal peut être commise dans le cas d'un hyper-réseau décoré (c'est-à-dire possédant plus d'un motif atomique par maille élémentaire). Cette mauvaise indexation empêche toute détermination correcte des motifs atomiques de la structure. Cet avertissement est illustré par un exemple pédagogique unidimensionnel et par un exemple bidimensionnel plus réaliste pouvant être pertinent pour décrire l'alliage décagonal AlNiCo.
\end{abstract}

\begin{abstract}
The diffraction pattern of quasicrystals can be misindexed in cases of decorated hyperlattices (more than one hyperspace atomic motif per a unit cell). The misindexing is fatal for proper determination of the atomic motifs. This warning is illustrated by a pedagogical $1 \mathrm{D}$ example and a more realistic $2 \mathrm{D}$ example, which might be related to the decagonal AlNiCo alloy.
\end{abstract}

\section{Introduction.}

Ten years after the discovery of the first quasicrystal [1], the basic question "Where are the atoms?" is, in general, still unanswered. This inability to solve atomic structures of quasicrystals is depressing especially in view of the fact that many attempts have been made, hundreds of quasicrystalline alloys have been synthesized and some of them are regarded as "perfect", like AlCuFe and AlPdMn [2]. Doubts about metastability and loose long range order have been thrown aside, long ago: quasicrystals were found to be thermodynamically stable (in certain regions of their alloy phase diagrams) and to exhibit true sharp Bragg diffraction peaks (correlation length estimates vary from $\approx 1 \mu \mathrm{m}$ [3] to nearly $1 \mathrm{~mm}$ [4], which is as good as in best periodic crystals; for a review see, say, $[5,6])$. We face a deterministic structure, with perfect

$\left({ }^{*}\right)$ Present address: Department of Physics, University of Wisconsin, Madison, WI 53706, U.S.A. 
long range order and high symmetry, but, in general, cannot solve it. We could count barely a couple of quasicrystalline, alloys whose structures have been deciphered to some reasonable extent.

On the one hand, this apparent inability to solve the structure is not so surprising: all standard crystallographic methods rely on periodicity and cannot be directly translated to the field of quasiperiodicity. On the other hand, a method to solve structures of quasicrystals, based on the hyperspace crystallography, was formulated several years ago. It was first applied by Gratias et al. [7] to AlMn and then further improved by Janot et al. [8] and de Boissieu et al. [9] by employing contrast variation technique which allows one to disentangle contributions from different atomic species by studying the neutron diffraction from samples with isotopic substitution.

The "first - order" models of the icosahedral AlCuLi [9, 10, 11], AlCuFe [12] and AlPdMn [13] as well as of the decagonal AlCuCo [14] and AlNiCo [15] were obtained by this method. They give valuable information about the atomic positions and reasonably agree with the diffraction data, but do not render the structure with the accuracy accepted in conventional crystallography. A large part of the difficulty should be attributed to the inferior quality of the samples which were available until three years ago: early samples contained a lot of disorder such as phason strain distribution, compositional inhomogeneity and chemical disorder. Nevertheless, even in the case of perfect sample quality, we point on certain peculiarities of the used method itself which might complicate the deciphering procedure.

Solving the structure of either a crystal or a quasicrystal is well-known to consist of three steps: (1) indexing experimentally obtained diffraction pattern (X-ray or neutron) and measuring peak intensities; (2) determining unmeasurable phases of the diffraction spots and (3) obtaining atomic coordinates from measured amplitudes and determined phases (Fourier synthesis). In conventional crystallography, steps 1 and 3 are classic and only step 2 makes a problem, which could be solved by well established methods. Not only fail these methods to work for quasicrystals, even steps 1 and 3 appear to be non-trivial in the quasiperiodic case. Indexing an experimentally obtained diffraction pattern of a quasicrystal is ambiguous due to the lack of the shortest reciprocal lattice vector. Fourier synthesis could be a mere formality, just performing a Fourier transform, only if one had the whole infinite series of amplitudes measured. In conventional crystallography this difficulty does not arise: a moderate number of peaks suffices to give the structure. In contrast, in quasicrystallography, truncating the Fourier series poses quite a problem [16]: atomic motifs (acceptance domains, projection windows) in the hyperspace emerge with smeared, rounded faces. If such a crude motif is used for getting atomic coordinates the result is unsatisfactory: atoms appear to split, unrealistic short and long spacings emerge, etc. This is why step 3 in quasicrystals includes a nontrivial procedure unknown in conventional crystallography: cutting experimentally obtained (round) motifs to polygons $[12,17,18,19,20]$. Only when such a polygonal motif is obtained could the structure be regarded as solved. (Of course, the diffraction pattern calculated from this motif should agree with the experimental one).

Despite the above argued nontriviality of steps 1 and 3 , little emphasis has been made on them up to now. Most of the researchers were overwhelmed with step 2, the phase problem, which is extremely complex and still unsolved in general. Not underestimating the difficulty and the importance of the phase problem, we want to issue a warning: mistakes could be naturally made in truncating motifs and even in indexing. Moreover, a mistake on the first, indexing stage, combined with truncation effects, could ruin the whole subsequent structure work, even if one knows how to determine all the phases. Accordingly, below we will concentrate on steps 1 and 3 only, as if somebody would know the way to solve the quasiperiodic phase problem (we wish we were such lucky people). 
We show below that carving polygons (step 3) goes relatively smoothly in quasicrystals with simple Bravais hyperlattices but could become virtually impossible in quasicrystals based on more complicated, decorated ones. This puts forth a question: if one has a finite set of experimentally measured peak intensities, how can one distinguish between a decorated and an undecorated lattice? The answer appears to be rather simple: collect enough weak spots and index them very carefully. Knowing a relatively small set (actual size depends on particularities) of strongest peaks could be insufficient for a correct determination of a decorated structure. Fortunately, the solution has nothing to do with solving the phase problem and involves only proper indexing. However, we argue that, in quasicrystals, failure to notice a superstructure could ruin all attempts to decipher a "parent" structure (if it is legible to refer to such a structure at all).

Our concern about complex quasilattices was prompted by the above mentioned inability to adequately determine the structure. Moreover, recently we became aware of the experiment by Edagawa et al. [22] who reported extremely weak superlattice peaks in well annealed decagonal AlNiCo, which was earlier viewed as undecorated $[14,15,20]$. If these results were to be confirmed, AlNiCo could become a real life example of what we are arguing about.

The main method we use in the present paper is a simulation of the experimental structure refinement. We introduce a model, i.e. place atoms in deterministic positions by specifically defining corresponding atomic motifs in the hyperspace, then we calculate the diffraction spectrum and truncate it at some threshold intensity (weak spots are thrown away as "undetectable"); this gives "experimental" spectrum. Then we act as if we forgot our model structure and knew only this "experimental" spectrum, trying to "solve" the structure within the framework of hyperspace crystallography, in the way it is usually done with real experimental diffraction spectra. Finally, we check if the obtained structure agrees with the original. To separate truncation and indexing effects from inaccuracies in phase determination we use exact phases from our model, simulating a person who can solve any phase problem. In real life difficulties and uncertainties of step 2 only aggravate the situation.

Our "deciphering simulation" is carried out four times: on a pair of 1D toy models followed by a pair of more realistic $2 D$ decagonal models. The latter are two binary tilings [23, 24], which are believed to be relevant to real AlNiCo and AlCuCo [20]. The models, both 1D and $2 \mathrm{D}$, are grouped in pairs, one in each pair has a simple undecorated Bravais lattice with just one hyper-atom per unit hyper-cell, the other possesses a decorated lattice, with several hyper-atoms in a unit hyper-cell.

\section{One-dimensional tilings.}

In this section we discuss two simple 1D structures derived from the Fibonacci sequence. They illustrate in a pedagogical way the points discussed in this paper since they contain the relevant ingredients but only need a two-dimensional hyperspace description. Both structures are tilings of the line by the two segments, $\mathrm{L}$ and $\mathrm{S}$ of respective lengths $l=\tau \equiv(1+\sqrt{5}) / 2$ (the golden mean) and $s=1$. The frequency ratio of the $\mathrm{L}$ and $\mathrm{S}$ tiles in both tilings is the same (equal to $\tau)$.

The first one is the usual 1D quasicrystal which corresponds to the substitution rules $\mathrm{L} \longrightarrow$ LS and $\mathrm{S} \longrightarrow \mathrm{L}$ and which can also be obtained by the strip and projection method from the square lattice $[25,26,27]$ with the lattice parameter of $\sqrt{\tau+2}$. Let us recall that in this $2 \mathrm{D}$ space one considers a line, $E^{\|}$, of slope $\tau^{-1}$ with respect to the square lattice, a perpendicular line, $E^{\perp}$, and a strip generated by shifting the square cell along $E^{\|}$. The tiling is the projection, onto $E^{\|}$, of the staircase made of the square edges included in this strip (Fig. 1a). The projection of the strip onto $E^{\perp}$ is an acceptance domain: among the vertices of 


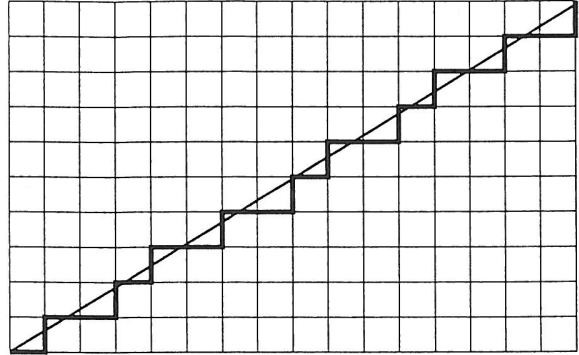

a)

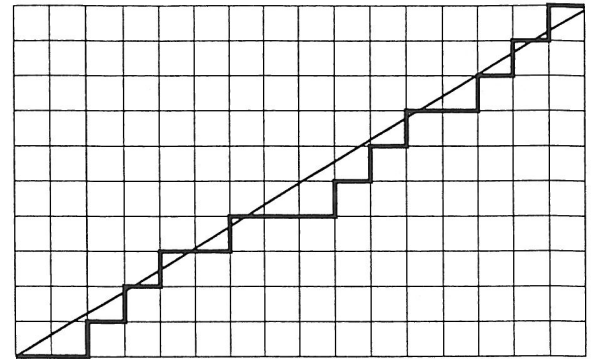

b)

Fig. 1. - Two-dimensional representations of the Fibonacci tiling (a) and the decorated tiling (b) by $\mathrm{L}$ and $\mathrm{S}$ segments. The $\mathrm{L}$ and $\mathrm{S}$ segments are represented by horizontal and vertical square edges, respectively

the square lattice, all those and only those which project onto $E^{\perp}$ in this domain correspond to the tiling vertices.

The second tiling is obtained by applying once the substitution rules $\mathrm{L} \longrightarrow$ LLSL and $\mathrm{S} \longrightarrow$ SLS on the first tiling: all original tiles have their length multiplied by a factor $\Theta=\tau+2$, and then, are decorated by these patterns (the limit of successive iterations of this substitution has been studied in $[28,29]$ ). Considering the same square lattice as before, the tiling is the projection onto $E^{\|}$of the staircase line shown in figure $1 \mathrm{~b}$. While the whole staircase line is contained in a strip of width $(3-\tau)$ times larger than the previous strip, not all the lattice points included in this strip project onto tiling vertices. Therefore the projection of this strip onto $E^{\perp}$ is not an acceptance domain and this 2D square lattice cannot be used to obtain the tiling within the framework of the strip and projection method. However we know that this tiling can be viewed as a decoration of the first one scaled by the factor $\Theta$ and thus it can be described using a square lattice $\Theta$ times larger. The decoration itself can be easily described in the 2D hyper-space by the oblique tiling method [30,31] or by the cut method $[32,33]$. In these methods the set of the tiling vertices is the intersection of $E^{\|}$with atomic motifs (or atomic surfaces or window functions) periodically set on the hyper-space lattice. The atomic motifs are the acceptance domains up to the inversion operation. The substitution rule used to decorate the inflated Fibonacci tiling can be directly applied in the $2 \mathrm{D}$ hyper-space as shown in figure $2 \mathrm{~b}$ : the original atomic motif which corresponds to the left vertex of a scaled $\mathrm{L}$ tile is substituted by four atomic motifs, identical but translated along $E^{\|}$by $0, \tau, 2 \tau, 2 \tau+1$; in the same way, the original atomic motif which corresponds to the left vertex of a scaled $\mathrm{S}$ tile is substituted by three atomic motifs, identical but translated by 0,1 and $1+\tau$.

Note that any scaling of the $2 \mathrm{D}$ crystal along $E^{\perp}$ also describes the same tiling since it does not change the intersection with $E^{l l}$. Therefore if we compress the structure along $E^{\perp}$ by a factor $\tau^{2}$, we obtain a valid representation with a $2 \mathrm{D}$ oblique crystal which is a super-lattice of the Fibonacci hyper-lattice (Fig. 2c) [34] since this lattice is related to the Fibonacci lattice by the deformation matrix

$$
M=\left(\begin{array}{ll}
3 & 1 \\
1 & 2
\end{array}\right)
$$

In this oblique crystal, the different atomic motifs lie on vertices of the Fibonacci $2 \mathrm{D}$ crystal. This oblique crystal has a super-cell 5 times larger and can be viewed as a chemically ordered 




a)

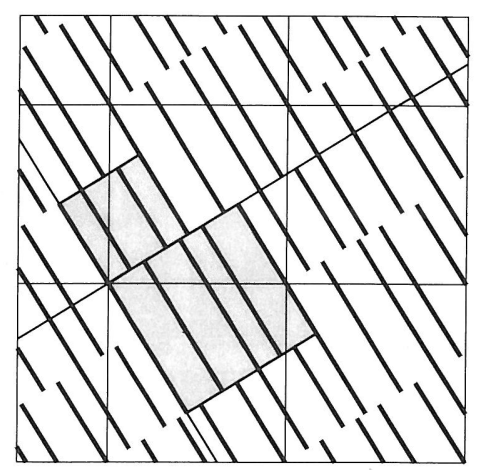

b)

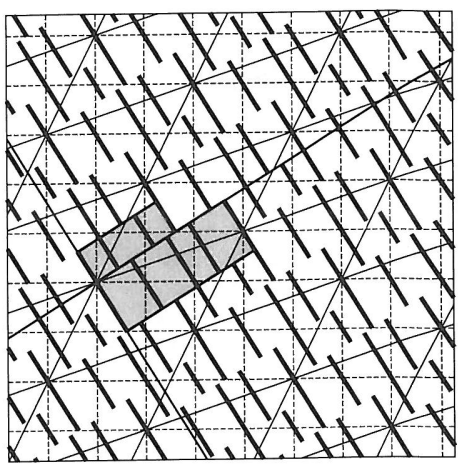

c)

Fig. 2. - Two-dimensional crystals which lead to the Fibonacci tiling (a) and the decorated tiling (b or c) by the cut method. The elementary $2 \mathrm{D}$ cells of the oblique tilings are also represented (in grey color). The parallel space L and S segments have, respectively, the same length in all cases.

Fibonacci crystal. In the following, we will describe the decorated tiling with the square lattice to keep the simplicity of orthogonal basis but both can equally be used.

Thus the two tilings are described in the 2D hyper-space by lattices with different parameters and unit cell contents. From the point of view of crystallography, we would like to distinguish these lattices using simulated diffraction data. We will show that the experimental resolution leads, of course, to uncertainties on the boundaries of the atomic motifs, but can also give a wrong unit cell description.

The $2 \mathrm{D}$ reciprocal-lattice vectors are

$$
\mathbf{Q}=\frac{2 \pi}{A}\left(n_{1} \mathbf{q}_{1}+n_{2} \mathrm{q}_{2}\right)
$$

where $\left(\mathbf{q}_{1}, \mathbf{q}_{\mathbf{2}}\right)$ is the canonical orthonormal basis of the reciprocal-space, $\left(n_{1}, n_{2}\right)$ is a pair of integer indices and $A$ is the lattice parameter.

The Fourier spectra of the tilings consist of Bragg peaks located on the projections of the $\mathbf{Q}$ vectors onto the reciprocal space $\widetilde{E}^{\|}: \mathbf{q} \equiv \mathbf{Q}^{\|}=k\left(n_{1} \tau+n_{2}\right)$, where the constant $k$ equals $k_{\text {fibo }}=2 \pi /(\tau+2)$ for the Fibonacci tiling and equals $k_{\text {deco }}=k_{\text {fibo }} / \Theta=k_{\text {fibo }}(3-\tau) / 5$ for the decorated tiling. Since $(3-\tau)\left(n_{1} \tau+n_{2}\right) / 5$ can be written as $\left(m_{1} \tau+m_{2}\right) / 5$, where $\left(m_{1}, m_{2}\right)$ is a new pair of integer indices, then all Fibonacci peak positions are also positions for the decorated structure. However, the converse is not true: only positions corresponding to $m_{1}$ and $m_{2}$ which are integer multiples of 5 are the positions of the Fibonacci tiling.

To get the amplitudes of the diffraction pattern, we have first to compute the Fourier transforms in the 2D hyper-space. Since the $2 \mathrm{D}$ crystals are square lattices convoluted by atomic motifs, their Fourier transforms are square lattices of delta functions multiplied by the form factors. Figure 3 shows the two form factors. The form factor in the Fibonacci case is constant along $\widetilde{E}^{\|}$, whereas interferences between atomic motifs in the decorated case lead to a more complex function. Since the lattice parameter of the latter 2D crystal is $\Theta$ times larger than that of the first crystal, its lattice parameter in reciprocal space is $\Theta$ times smaller. However, as shown in figure $4 \mathrm{~b}$, the peak intensities do not follow the usual $\mathbf{Q}^{\perp}$ decrease along $\widetilde{E}^{\perp}$ (cf. Fig. 4a) because of the shape of the form factor. Indeed, the most intense peaks in the $2 D$ reciprocal space are projected onto the same positions in $\widetilde{E}^{\|}$for the two tilings. 


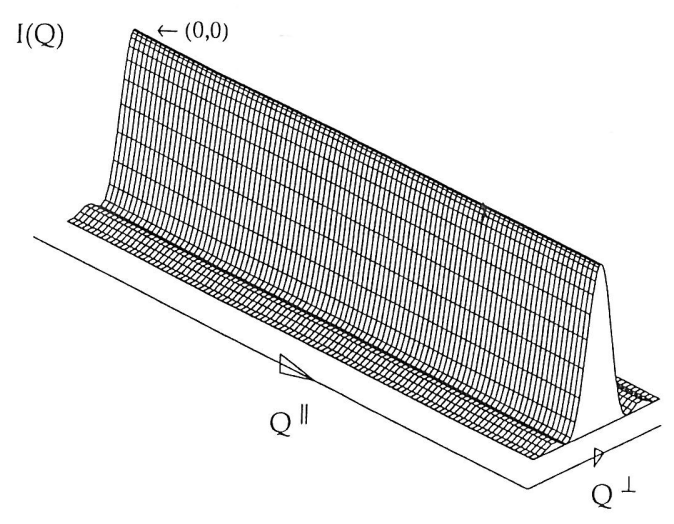

a)

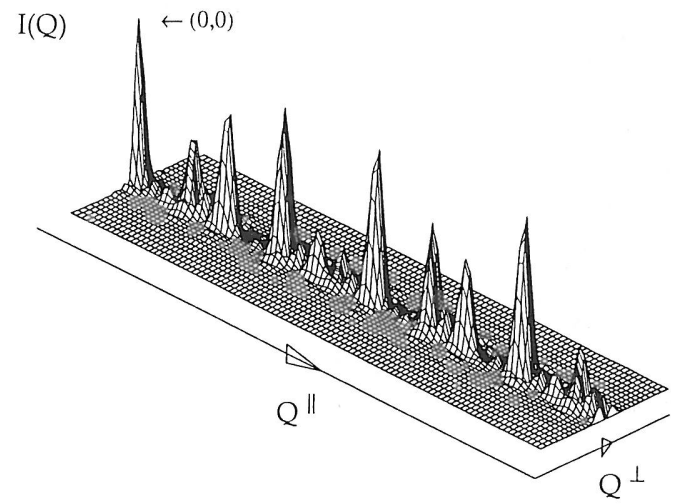

b)

Fig. 3. - Form factor intensities of the atomic motifs of the Fibonacci tiling (a) and the decorated tiling (b). The two plots are drawn at the same scale.

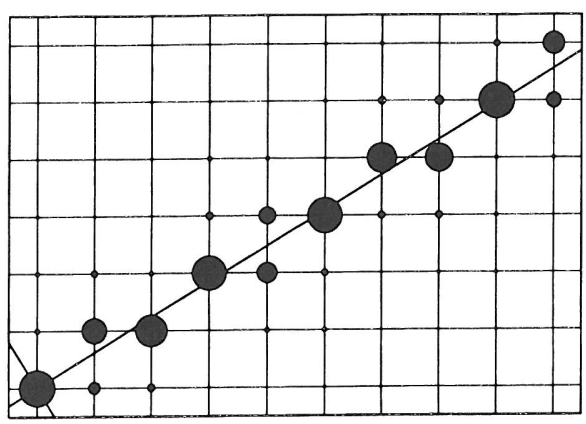

a)

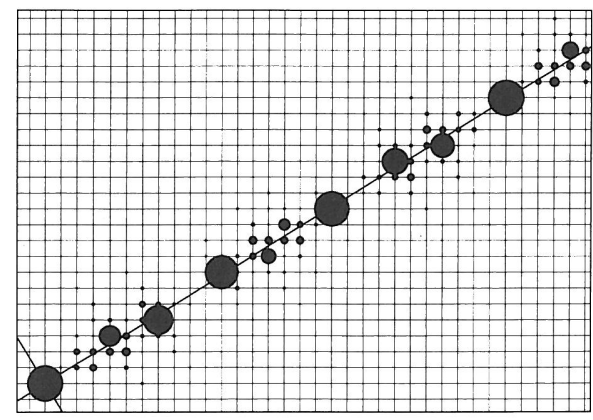

b)

Fig. 4. - Diffraction patterns of the two-dimensional crystals shown in figure 2 and corresponding to the Fibonacci tiling (a) and the decorated tiling (b). The Bragg peaks are set on the nodes of the square reciprocal lattices. The disk areas are proportional to the intensities. The reciprocal length scale is the same for the two figures (corresponding to the same tile lengths in real space $E^{\|}$).

The diffraction patterns of the two tilings shown in figure 5 are different but similar, and the extra peaks which exist in the decorated tiling are not the main peaks. Their intensities have been found to be less than or equal to $6 \%$ of the maximum intensity.

Now, if one neglects these weak peaks, one would be able to index all the stronger peaks of the decorated tiling diffraction pattern with a $2 \mathrm{D}$ lattice parameter $A$ corresponding to the Fibonacci tiling, i.e. $\Theta$ times smaller than the correct parameter. The weaker peaks could still be approximately indexed using large index values. If we proceed in this wrong way, what atomic motifs would we get? Neglecting all the (weak) peaks which cannot be exactly indexed in the incorrect basis, lifting the others in the $2 \mathrm{D}$ cubic lattice of parameter $2 \pi / \sqrt{\tau+2}$ and performing an inverse Fourier transform, we do not get window function ( 1 inside; 0 outside), but a superposition of the actual atomic motifs set periodically on the wrong lattice. The profile of the function obtained is shown in figure 6 as well as the profile obtained if we take into account the rounding errors due to the $\mathbf{Q}$ truncation effect $\left(q_{\max }=2 \pi \times 8.1 / s\right.$ where $s$ is 

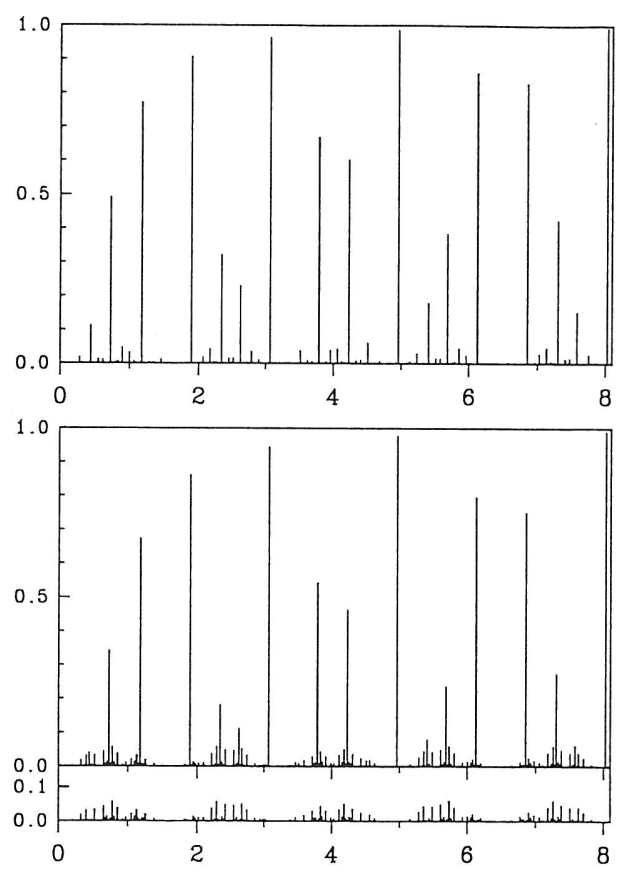

Fig. 5. - Diffraction patterns of the Fibonacci tiling (above) and the decorated tiling (below) (with a unit mass set at the left extremity of each of the tiles). The peaks which have no counterpart in the Fibonacci tiling (superlattice peaks) are shown below the total curve of the decorated tiling.

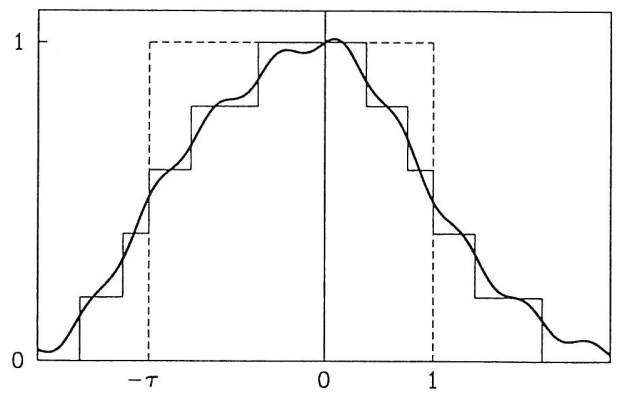

Fig. 6. - Cross-section along $E^{\perp}$ of the two-dimensional crystal obtained by inverse Fourier transform from the decorated tiling diffraction pattern using an incorrect peak indexing. Thin line: without truncation effect; thick line: with intensity and $q$-range truncation effects; dash line: cross-section of the Fibonacci atomic motif indicating the scales.

the length of the $\mathrm{S}$ segments $)$ and a low intensity cut-off $\left(I_{\min }=0.001 \times I(0)\right)$. For practical purpose a cut-off along $\widetilde{E}^{\perp}\left(q_{\max }^{\perp}=2 \pi \times 8.3 / s\right)$ has been used but was completely overcome by the intensity cut-off.

Taking into account these cut-off effects, figure 7 shows the "experimental" $2 \mathrm{D}$ atomic densities obtained by inverse Fourier transform of the Fourier amplitude which is exactly known here. While the structure is resolved in figure $7 \mathrm{a}$ where the correct indexing has been done, figure $7 \mathrm{~b}$ shows that we loose the correct hyper-lattice periodicity when we neglect the weak 


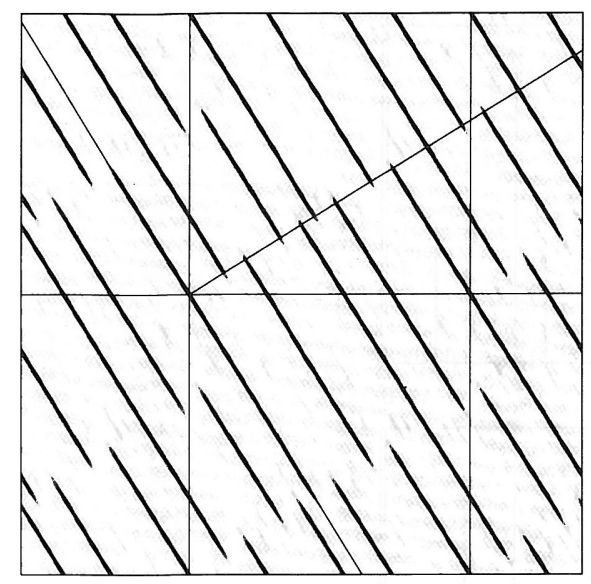

a)

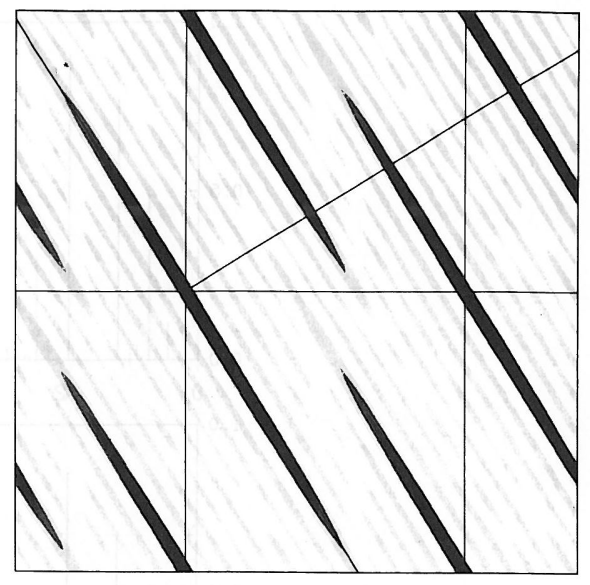

b)

Fig. 7. - Density plots of the inverse Fourier Transform obtained from the decorated tiling diffraction pattern using the correct peak indexing (a) and the incorrect indexing (b) when lifting the diffraction peaks in the reciprocal hyper-space. Compare the left plot with the original $2 \mathrm{D}$-crystal in figure $2 \mathrm{~b}$. The scale of the right plot is $\tau+2$ times larger than the scale of the left one.

peaks. The comparison with figure $2 \mathrm{c}$ shows that we get a mean atomic density distribution instead of the different atomic motifs of the super-lattice cell. An attempt to extract a sharp window function from this density distribution would lead to the Fibonacci tiling.

The correct indexing of the weak peaks may be achieved by looking at their $\mathbf{Q}^{\perp}$ dependence. Any attempt to index these peaks in the wrong basis will lead to very high $\mathbf{Q}^{\perp}$ reciprocal vectors, which are inconsistent with a general $\mathbf{Q}^{\perp}$ decrease of the intensity.

\section{Decagonal binary tilings.}

The two $2 D$ models to be discussed below are rather similar to the above $1 D$ toy models in their ideology, but could be more relevant to real decagonal alloys, namely AlNiCo and $\mathrm{AlCuCo}$ [14, $20,21]$. However, to avoid obscuring physics by crystallography we restrict ourselves to a cluster model, without specifying where real $\mathrm{Al}, \mathrm{Cu}$ and $\mathrm{Co}$ atoms are. We also neglect the 3D structure of real decagonal quasicrystals, which are periodic along the ten-fold axis and may have more than one atomic layer per vertical period [14, 20]. Accordingly, our models are purely two-dimensional, built of flat "atoms". As in the preceding $1 \mathrm{D}$ example, we compare two quasicrystals: one based on a simple quasilattice, the other on a decorated one. Both belong to a wide class of so-called decagonal binary tilings. One may recall a definition [24]. Consider a tiling of a plane by the two Penrose tiles (a thin rhombus with $\pi / 5$ and $4 \pi / 5$ angles and a fat rhombus with $2 \pi / 5$ and $3 \pi / 5$ angles).

Definition 1. A vertex of a tiling is called even (odd) if all angles made by tiles meeting at this vertex are of the form $N \pi / 5$, where $N$ is even (odd). A tiling of a plane by the two Penrose tiles such that any vertex is either even or odd is called a binary tiling. Odd vertices are decorated with "large atoms", even vertices with "small atoms".

In real materials large and small "atoms" are pretty large clusters, whose decoration may vary from one alloy to another. An example of such a decoration for $\mathrm{AlCuCo}$ can be found in [20]. Though restrictions imposed by Definition 1 resemble a sort of matching rules, these 
rules appear to be not binding (unlike the famous Penrose arrow rules): there exist infinitely many binary tilings, there are even random tilings among them [35, 24, 6, 36]. However, both our models to consider are $100 \%$ deterministic (as usual, up to a uniform phason shift). To insure this perfect quasiperiodicity one must impose matching restrictions stronger than in Definition 1. Though the existence of so-called strong local rules is an important property [37, 38], it is absolutely irrelevant to what follows; all we need is a given deterministic quasiperiodic structure defined by the cut (or, equivalently, projection) method. No one cares about why it is such, in this study.

Before defining the two models, let us recall some well-known general properties of binary tilings. Any tiling of the plane $E_{\|}^{2}$ by the two Penrose tiles, can be lifted to a five-dimensional space in the standard manner $[6,25,26,27]$. Any vertex of the tiling can be presented in the form

$$
\mathbf{R}=\sum_{j=1}^{5} m_{j} \mathbf{b}_{j}^{\|}
$$

where $\mathbf{b}_{j}^{\|}$denotes five vectors directed along the tile sides ( $\mathbf{b}_{j}^{\|}$form a regular 5 -star) and $m_{j}$ are five integers. Since the sum of five vectors $\mathbf{b}_{j}^{\|}$is zero, the five components $m_{j}$ are defined up to a common additive integer and we can consider only those such that $m_{\Delta}=\sum_{j=1}^{5} m_{j}$ belongs to $\{-2,-1,0,1,2\}$. Accordingly, any vertex of a tiling is mapped unambiguously onto a point of $\mathbb{Z}^{5}$ and is associated with a vertex of a $5 \mathrm{D}$ hyper-cubic lattice defined by its basis $\left(\mathbf{b}_{1}, \mathbf{b}_{2}, \mathbf{b}_{3}, \mathbf{b}_{4}, \mathbf{b}_{5}\right)$ such that the projections of the vectors $\mathbf{b}_{j}$ onto $E_{\|}^{2}$ are $\mathbf{b}_{j}^{\|}$. The $5 \mathrm{D}$-points lifted up in this way have components in the $3 \mathrm{D}$ space, $E_{\perp}^{3}$, perpendicular to $E_{\|}^{2}$. A $5 \mathrm{D}$-integer vector, $\mathbf{B}$, equal to $(1,1,1,1,1)$ in the $\mathbf{b}_{j}$-basis, belongs to $E_{\perp}^{3}$ since its projection onto $E_{\|}^{2}$ is zero. This means that the projections of vertices onto $E_{\perp}^{3}$ cannot fill 3D-domains densely, but are situated on layers perpendicular to $\mathbf{B}$. These layers are indexed by $m_{\Delta}$ which can take a maximum of five values. However, for any binary tiling only 3 layers are occupied:

Assertion 1. All odd vertices (large atoms) of a binary tiling project onto layer zero, all even vertices (small atoms) onto layers \pm 1 (the origin being chosen at an odd vertex).

Note that this assertion holds for any binary tiling, not necessarily obtained with the cut method; it is valid even for random tilings which do not show any acceptance domain. The property is easily derivable from Definition $1[35,36]$

Since we have adopted a $5 \mathrm{D}$ presentation (some people prefer an equivalent $4 \mathrm{D}$ language [6]) the atomic motifs are $3 \mathrm{D}$ objects embedded in $E_{\perp}^{3}$, consisting of at most five flat polygons in the five layers perpendicular to $\mathbf{B}$. Accordingly, the well-known four pentagons of the standard Penrose tiling [39] will be referred to as one atomic motif.

We now describe the two quasicrystalline binary tilings studied in this paper and shown in figure 8:

1) A simple decagonal quasicrystal is defined by the standard cut method with one motif consisting of three polygons shown in figure 10: a decagon $[20,37]$ which corresponds to the large atoms and two 5-stars which correspond to the small ones. This atomic motif is set periodically on the vertices of the $5 \mathrm{D}$ cubic lattice defined by the $\mathbf{b}_{j}$-basis. The tiling obtained is related to the triangle pattern defined in $[37,40]$. It was argued to be relevant to real $\mathrm{AlCuCo}$ [20].

2) A decorated decagonal quasicrystal is obtained by decorating standard Penrose tiling: it is the type II tiling of [24]. The tile edges of the initial Penrose tiling are five vectors $\mathbf{e}_{j}^{\|}$forming a five-fold star. The vectors $\mathbf{b}_{j}^{\|}$(corresponding to the binary tiling edges) are deduced from the 


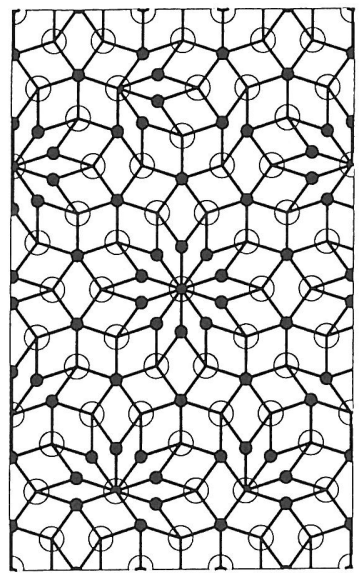

a)

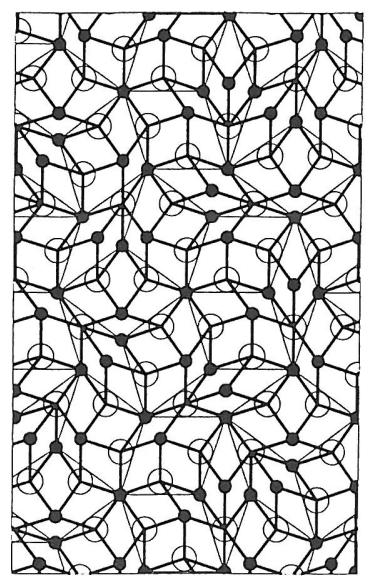

b)

Fig. 8. - Two binary tilings: a) simple decagonal, b) decorated decagonal quasicrystals (the underlying Penrose tiling is drawn with thin lines). Large and small particles are set on the two vertex types.
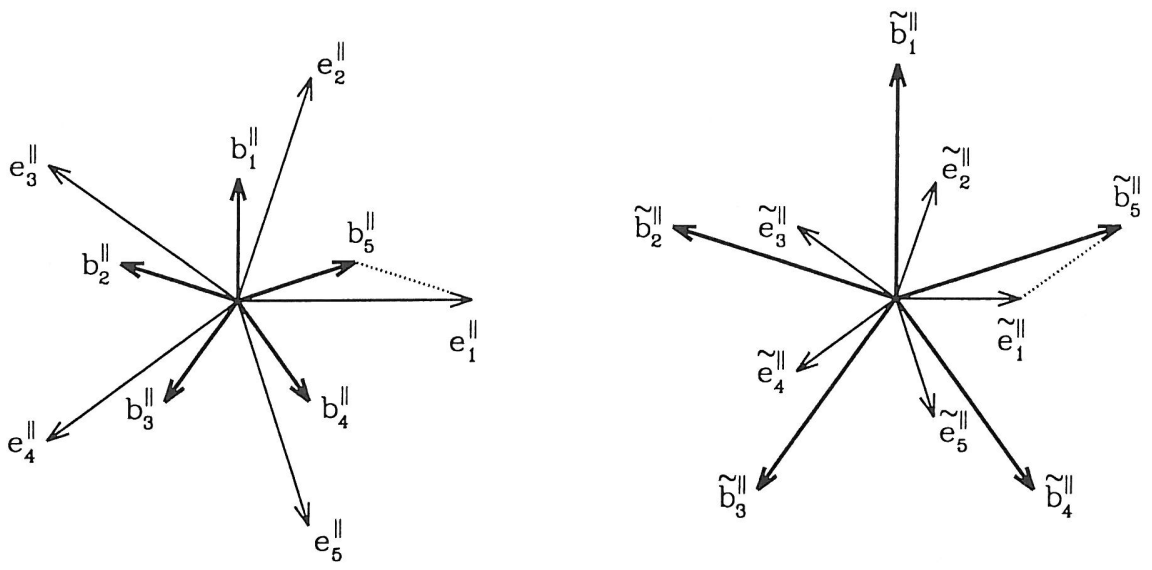

Fig. 9. - Parallel projection of Penrose and binary bases. (a: left) direct space: $\mathbf{e}_{j}^{\|}$(Penrose) and $\mathbf{b}_{j}^{\|}$(binary), (b: right) reciprocal space: $\widetilde{\mathbf{e}}_{j}^{\|}$(Penrose) and $\widetilde{\mathbf{b}}_{j}^{\|}$(binary).

vectors $\mathbf{e}_{j}^{\|}$by a rotation of $\pi / 2$ and a length scaling down of $\theta=\sqrt{\tau+2}$ (see Fig. 9a; the two five-fold stars are globally rotated by a $\pi / 10(\bmod 2 \pi / 5)$ angle, and we choose $\pi / 2$ for better indexing harmony). The non-quasicrystalline structure obtained after an infinite number of successive decorations has been studied in [41,28], but here we consider the quasicrystalline tiling obtained from the Penrose tiling after only one iteration. Calling $\mathrm{L}$ and $\mathrm{S}$ the obtuse and acute tiles respectively, we see that this tiling is the $2 \mathrm{D}$ analog of the $1 \mathrm{D}$ decorated tiling studied in section 1 : both are decorated with the rules L $\rightarrow$ LLSL and S $\rightarrow$ SLS. The 1D-tile length scale factor $\Theta$ is equal to the 2D-tile area scale factor $\theta^{2}$.

Note that both tilings are $100 \%$ deterministic, since they are obtained by the cut method, followed in the second case by a deterministic decoration. Both tilings are also made with the 


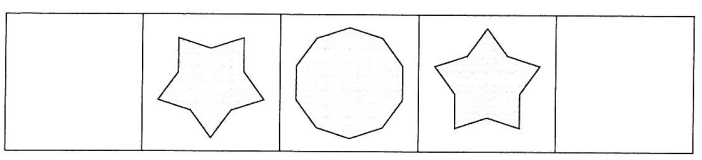

Fig. 10. - Hyperspace description of the simple decagonal quasicrystal in the $\mathbf{b}_{j}$ cubic lattice: the atomic motif layers are indexed by $m_{\Delta}=-1,0,1$ (from the left to the right).

same Penrose tiles $\mathrm{L}$ and $\mathrm{S}$, with the same edge length $b=\left\|\mathbf{b}_{j}^{\|}\right\|$and, by construction, with the same five-fold edge orientation. The frequency ratio of the $L$ and $\mathrm{S}$ tiles is equal to $\tau$ in both tilings [24].

Assertion 1 holds for the second, decorated tiling since it is a binary tiling, i.e. the vertices lifted to the cubic lattice defined by the $\mathbf{b}_{j}$-basis project onto only three layers in $E_{\perp}^{3}$. However, vectors $\mathbf{b}_{j}$ 's do not form a proper lattice basis for the decorated tiling. The proper basis is the basis $\left(\mathbf{e}_{1}, \mathbf{e}_{2}, \mathbf{e}_{3}, \mathbf{e}_{4}, \mathbf{e}_{5}\right)$, used to build the initial Penrose tiling.

The $5 \mathrm{D}$ orthogonal $\mathbf{e}_{j}$-basis is related to the $5 \mathrm{D}$ orthogonal $\mathbf{b}_{j}$-basis by a scaling of factor $\theta$ and a rotation of $\pi / 2$ which let globally invariant the spaces $E_{\|}^{2}, E_{\perp}^{2}$ and $\Delta_{\perp}$ (where $\Delta_{\perp}$ is the $1 \mathrm{D}$ space generated by the diagonal vector $\mathbf{B}$ and $E_{\perp}^{2}$ is its orthogonal supplementary space in $\left.E_{\perp}^{3}\right)$. Thus we have the following relations between the projections of $\mathbf{e}_{j}$ and $\mathrm{b}_{j}$ onto $E_{\|}^{2}, E_{\perp}^{2}$ and $\Delta_{\perp}$ :

$$
\begin{aligned}
\mathbf{e}_{j}^{\|} & =\mathbf{b}_{j-1}^{\|}-\mathbf{b}_{j+1}^{\|} \\
\mathbf{e}_{j}^{\perp} & =\tau\left(\mathbf{b}_{j-1}^{\perp}-\mathbf{b}_{j+1}^{\perp}\right)=\mathbf{b}_{j+2}^{\perp}-\mathbf{b}_{j-2}^{\perp} \\
\mathbf{e}_{j}^{\Delta} & =\theta \mathbf{b}_{j}^{\Delta} \\
\mathbf{b}_{j}^{\|} & =\frac{1}{5}\left[2\left(\mathbf{e}_{j+1}^{\|}-\mathbf{e}_{j-1}^{\|}\right)+\left(\mathbf{e}_{j-2}^{\|}-\mathbf{e}_{j+2}^{\|}\right)\right]
\end{aligned}
$$

Since the second binary tiling is built by decorating the Penrose tiling it is quite natural to expect a decorated hyperlattice. Indeed, $1 / 5$ factor in equation (6) shows that not every vertex of the binary tiling acquires integer coordinates when lifted up with the $\mathbf{e}_{j}$-basis. In the classical cut method language this means the presence of more than one atomic motif per $5 \mathrm{D}$ unit cell. The structure can also be described as a super-lattice of the cubic $\mathrm{b}_{j}$-lattice if, instead of the cubic $\mathbf{e}_{j}$-lattice, we use a rhombohedral lattice with a super-cell 25 times larger than the $\mathbf{b}_{j}$-cell and defined by the basis $\left(\epsilon_{j}\right)$ given by

$$
\epsilon_{j}=\mathbf{b}_{j-1}-\mathbf{b}_{j+1}+\mathbf{b}_{1}+\mathbf{b}_{2}+\mathbf{b}_{3}+\mathbf{b}_{4}+\mathbf{b}_{5}
$$

The $\epsilon_{j}$-lattice corresponds simply to a shear of the $\mathbf{e}_{j}$-lattice, scaling the perpendicular space while leaving the parallel space undisturbed. Thus equations (3) and (6) are the same with $\epsilon_{j}$ instead of $\mathbf{e}_{j}$ while equations (4) and (5) are replaced by

$$
\begin{aligned}
\epsilon_{j}^{\perp} & =\left(\mathbf{b}_{j-1}^{\perp}-\mathbf{b}_{j+1}^{\perp}\right) \\
\epsilon_{j}^{\Delta} & =5 \mathbf{b}_{j}^{\Delta}
\end{aligned}
$$

To describe the decorated tiling completely we have now to specify the unit cell content. The atomic motifs (in the Penrose $\mathbf{e}_{j}$-basis) are shown in figure 11 . They can be routinely deduced 

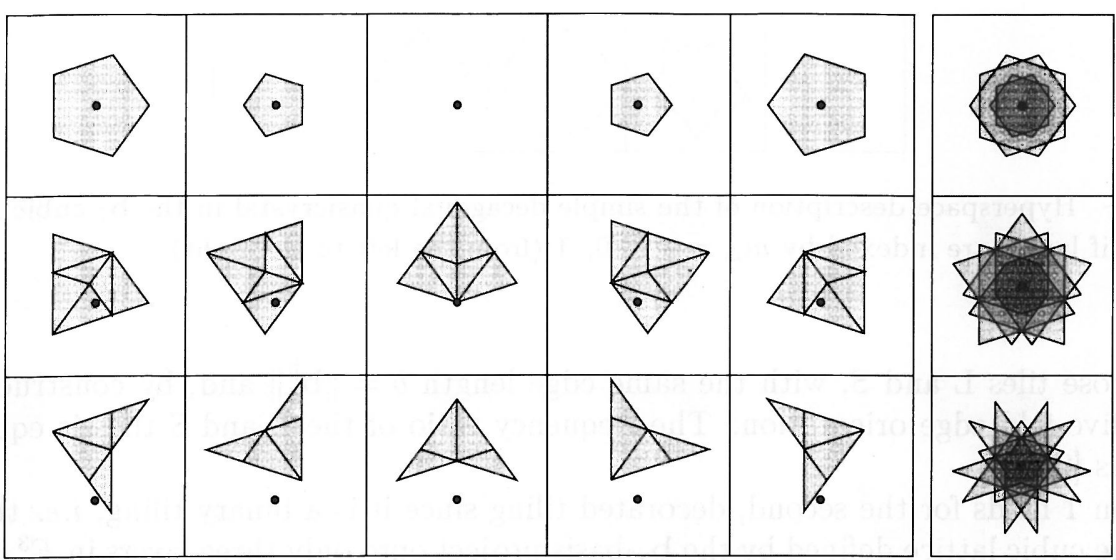

Fig. 11. - Hyperspace description of the decorated quasicrystal. The grey levels are obtained by plotting projections of the lifted tiling vertices. A black disk is set on each plane origin. (a: left) In the (proper) Penrose $\mathbf{e}_{j}$-lattice, the three atomic motifs, indexed by $\varepsilon=0,1,2$, are embedded in the spaces $\varepsilon \mathbf{b}_{1}^{\|}+E_{\perp}^{3}$ (respectively first, second and third lines). The layers are indexed by $n_{\Delta}=-2, \ldots, 2$ (from left to right). The acute and obtuse Robinson triangles define domains corresponding to the decoration of the large and sharp Penrose rhombs, respectively. In the (proper) $\epsilon_{j}$-lattice, the figures are the same with a scale $\tau$ times larger. (b: right) Result of an erroneous lifting of the decorated quasicrystal with (improper) binary $\mathbf{b}_{j}$-lattice: one gets regions of non constant density in three layers indexed by $m_{\Delta}=\varepsilon=0,1,2$ (from up to down) and embedded in the space $E_{\perp}^{3}$. The scale of the right plot is $\tau$ times larger than the scale of the left one.

from the known motifs of the Penrose tiling [39] and the rules of decoration [24]. The first motif is responsible for small atoms sitting on the sites of the parent Penrose tiling (Fig. 8b); this is simply the well-known motif of the standard Penrose tiling, consisting of four pentagons [39]. The second and third motifs describe, respectively, extra large and small atoms inserted inside the Penrose tiles (Fig. 8b).

The shape of the second motif is derived by the following procedure. First, note that according to the decoration rules [24] a large atom is placed near any bond of the parent Penrose tiling. Namely, the bond connecting the two Penrose sites at $\mathbf{X}^{\|}$and $\mathbf{X}^{\|}+\mathbf{e}_{j}^{\|}$gives rise to a large atom at $\mathbf{X}^{\|}+\mathbf{b}_{j-1}^{\|}$(Fig. 8b). Accordingly, we find a subset of the original standard Penrose motif describing points $\mathbf{X}$ such that $\mathbf{X}^{\perp}$ and $\mathbf{X}^{\perp}+\mathbf{e}_{j}^{\perp}$ both belong to the Penrose motif. Finally, the obtained submotifs associated with $\mathbf{e}_{j}$ are translated in parallel space by $\mathbf{b}_{j-1}^{\|}$. This has to be done for $j=1$ to 5 and at this latter stage it is useful to realize that the differences of any two of the five $\mathbf{b}_{j}^{\|}$vectors are integer linear combinations of the $\mathbf{e}_{j}^{\| \text {'s }}$ (Eqs. (6), (3)). Therefore, taking into account the periodicity of the $\mathbf{e}_{j}$-cubic lattice, all the fractional shifts by any of the five $\mathbf{b}_{j}^{\|}$'s can be reduced to only one fractional shift, say, along $\mathbf{b}_{1}^{\|}$. This gives a unique motif for all large atoms, which is displaced by $\mathbf{b}_{1}^{\|}$with respect to the parent Penrose motif, i.e., it is not embedded in $E_{\perp}^{3}$ but in $\mathbf{b}_{1}^{\|}+E_{\perp}^{3}$. The result is shown in figure 11 , second line.

The motif for extra small atoms (which appears to be unique on the same grounds) is 
obtained by similar considerations and shown in figure 11, third line; the displacement with respect to the parent Penrose motif is $2 \mathbf{b}_{1}^{\|}$, i.e., it is embedded in $2 \mathbf{b}_{1}^{\|}+E_{\perp}^{3}$.

The above findings could be summarized as

Assertion 2. Decorated decagonal quasicrystal (\#2) can be obtained by the cut method in the Penrose $\mathbf{e}_{j}$-lattice with three atomic motifs (14 polygons altogether) shown in figure 11.

However, if one does not recognize the super lattice periodicity, it is still possible to lift (improperly) the tiling up in the $5 \mathrm{D}$ cubic $\mathbf{b}_{j}$-lattice since the vertices satisfy equation (2). The lifted points project onto $E_{\perp}^{3}$ in domains located in only three layers. What do these domains look like? To answer this question let us compare lifting procedures in the Penrose $\mathbf{e}_{j}$-lattice and the binary $\mathbf{b}_{j}$-lattice.

In the former the coordinates of an arbitrary atom at a point $\mathbf{R}$ should be presented as

$$
\mathbf{R}=\varepsilon \mathbf{b}_{1}^{\|}+\sum_{j} n_{j} \mathbf{e}_{j}^{\|}=\varepsilon \mathbf{b}_{1}^{\|}+\sum_{j} n_{j} \mathbf{e}_{j}-\sum_{j} n_{j} \mathbf{e}_{j}^{\perp}-n_{\Delta} \mathbf{E} / 5
$$

where $\varepsilon$ is either 0,1 or 2 , and indexes the three atomic motifs. The sum $n_{\Delta}=\sum_{j} n_{j}$ indexes the five layers perpendicular to the diagonal vector $\mathbb{E}=\sum_{j} \mathbf{e}_{j}$. The vertex $\mathbf{R}$ is lifted to the point $\varepsilon \mathbf{b}_{1}^{\|}+\sum_{j} n_{j} \mathbf{e}_{j}$ which projects onto $E_{\perp}^{3}$ on the point $\sum_{j} n_{j} \mathbf{e}_{j}^{\perp}+n_{\Delta} \mathbf{E} / 5$, within one of the five layers, depending on $n_{\Delta}$.

In the binary basis the same point $\mathbb{R}$ can be written as

$$
\begin{aligned}
& \mathbf{R}=\varepsilon \mathbf{b}_{1}^{\|}+\sum_{j} n_{j} \mathbf{e}_{j}^{\|}=\varepsilon \mathbf{b}_{1}^{\|}+\sum_{j} n_{j}\left(\mathbf{b}_{j-1}^{\|}-\mathbf{b}_{j+1}^{\|}\right) \\
& =\varepsilon \mathbf{b}_{1}+\sum_{j} n_{j}\left(\mathbf{b}_{j-1}-\mathbf{b}_{j+1}\right)-\varepsilon \mathbf{b}_{1}^{\perp}-\sum_{j} n_{j}\left(\mathbf{b}_{j-1}^{\perp}-\mathbf{b}_{j+1}^{\perp}\right)-\varepsilon \mathbf{b}_{1}^{\Delta} \\
& =\mathbf{M}-\varepsilon \mathbf{b}_{1}^{\perp}-\tau^{-1} \sum_{j} n_{j} \mathbf{e}_{j}^{\perp}-\varepsilon \mathbf{B} / 5
\end{aligned}
$$

where $\mathbf{M}$ is a vertex of the $\mathbf{b}_{j}$-cubic lattice and where we have used equations (3) and (4). Thus vertex $\mathbb{R}$ is lifted to $\mathbf{M}$ which projects onto $E_{\perp}^{2}$ on the same point as before but scaled by the factor $\tau^{-1}$ and shifted by $\varepsilon \mathbf{b}_{1}^{\perp}$. Perpendicularly to the diagonal vector $\mathbf{B}, \mathbf{M}$ projects in three layers according to the value of $\varepsilon$. Thus in the space $E_{\perp}^{3}$, the binary tiling property (Ass. 1) is verified but the information about the five initial layers (given by $n_{\Delta}$ ) is missing: these five layers are merged together as shown in figure 11b. Accordingly, the density of points within the three domains in perpendicular space is not uniform and correct physical coordinates cannot be obtained by a simple cut from such domains. Only the use of Penrose $\mathbf{e}_{j}$-lattice (or a lattice deduced by a scaling in perpendicular space and giving an equivalent description as the $\epsilon_{j}$-lattice) secures against this mistake.

Below we proceed to the main question: how can one distinguish the two model systems having their diffraction patterns in hand? The two patterns are rather similar: both display ten-fold symmetry, true sharp Bragg peaks filling the reciprocal space everywhere densely, no diffuse scattering and positions of all spots can be indexed with five integers. Let us call $\left(\widetilde{\mathbf{b}}_{j}\right)$ the reciprocal vectors of the $\mathbf{b}_{j}$-cubic lattice associated with the simple lattice $(\# 1)$ and $\left(\widetilde{\mathbf{e}}_{j}\right)$ the reciprocal vectors of the $\mathbf{e}_{j}$-cubic lattice associated with the decorated lattice (\#2). The respective lengths of their parallel projections are $(2 / 5)(2 \pi / d)$, where $d$ is set to the length $b$ of the binary tiling bonds in the first model but to the length $e$ of the Penrose bonds in the second one. Thus $\left\|\widetilde{\mathbf{b}}_{j}^{\|}\right\|=\theta\left\|\widetilde{\mathbf{e}}_{j}^{\|}\right\|$(Fig. 9b).

There is no difficulty with the simple lattice (\#1): all peaks are given by a linear combination of the $\widetilde{\mathbf{b}}_{j}^{\|}$vectors, with the intensities obtained by Fourier transforming the unique motif of figure 10. The decorated quasicrystal (\#2), however, displays more peaks, since the reflections are generated by shorter reciprocal vectors $\widetilde{\mathbf{e}}_{j}^{\|}$. How do the diffraction patterns of the two 
models differ from each other? The bases are related in a way similar to equations (3) and (6):

$$
\begin{aligned}
\tilde{\mathbf{b}}_{j}^{\|} & =\widetilde{\mathbf{e}}_{j+1}^{\|}-\widetilde{\mathbf{e}}_{j-1}^{\|} \\
\widetilde{\mathbf{e}}_{j}^{\|} & =\frac{1}{5}\left[2\left(\widetilde{\mathbf{b}}_{j-1}^{\|}-\widetilde{\mathbf{b}}_{j+1}^{\|}\right)+\left(\widetilde{\mathbf{b}}_{j+2}^{\|}-\widetilde{\mathbf{b}}_{j-2}^{\|}\right)\right]
\end{aligned}
$$

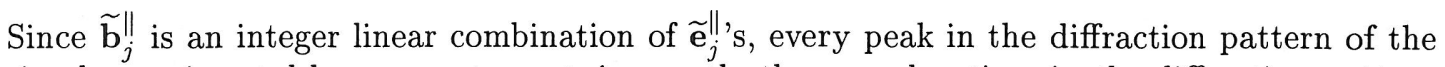
simple quasicrystal has a counterpart, in exactly the same location, in the diffraction pattern of the decorated quasicrystal. Because of the $1 / 5$ factor in equation (13) the converse is not true: not every reflection of model \#2 has a match in the model \#1.

Assertion 3. A diffraction spot of the decorated quasicrystal at $\mathbf{Q}^{\|}=\sum_{j} Q_{j} \widetilde{\mathbf{e}}_{j}^{\|}$has a counterpart in the diffraction pattern of the simple undecorated quasicrystal if and only if $Q_{\widetilde{\Delta}} \equiv \sum_{j} Q_{j}=0 \quad(\bmod 5)$.

$<<$ To prove this statement we have to relate the indices in the $\widetilde{\mathbf{e}}_{j}$ and $\widetilde{\mathbf{b}}_{j}$-bases: $\mathbf{Q}^{\|}=$ $\sum_{j} Q_{j} \widetilde{\mathbf{e}}_{j}^{\|}=\sum_{j} f_{j} \widetilde{\mathbf{b}}_{j}^{\|}$(note that the $f_{j}$ are not necessarily integer). Using equation (12) this relation means that there exists a constant $c$ such that $Q_{j}-c=f_{j-1}-f_{j+1}$ (recall the linear dependence $\sum_{j} \widetilde{\mathbf{e}}_{j}^{\|}=0$ ). Performing summation over $j$ defines the constant $c$, and thus

$$
f_{j-1}-f_{j+1}=Q_{j}-Q_{\widetilde{\Delta}} / 5
$$

Therefore, the spots can be indexed with integer $f_{j}$ coefficients only if $Q_{\widetilde{\Delta}}=0$. Conversely, we express the $\widetilde{\mathbf{e}}_{j}^{\|}$vectors through $\widetilde{\mathbf{e}}_{1}^{\|}$and $\widetilde{\mathbf{b}}_{j}^{\|}$vectors using equation (12) and thus

$$
\mathbf{Q}^{\|}=Q_{\widetilde{\Delta}} \widetilde{\mathbf{e}}_{1}^{\|}+\left(Q_{3}+Q_{5}\right) \widetilde{\mathbf{b}}_{2}^{\|}-Q_{2} \widetilde{\mathbf{b}}_{3}^{\|}+Q_{5} \widetilde{\mathbf{b}}_{4}^{\|}-\left(Q_{2}+Q_{4}\right) \widetilde{\mathbf{b}}_{5}^{\|}
$$

Therefore if $Q_{\widetilde{\Delta}}=0$ then $\mathbf{Q}^{\|}$is explicitly presented by equation (15) as $\mathbf{Q}^{\|}=\sum_{j} f_{j} \widetilde{\mathbf{b}}_{j}^{\|}$, where the coefficients $f_{j}$ are integer. $>>$

Equation (15) also allows a natural classification of peaks in the diffraction pattern \#2 (decorated structure): there are five families, classified by $Q_{\widetilde{\Delta}}$, within each family the peaks could be indexed in the binary $\widetilde{\mathbf{b}}_{j}$-basis, but the families are displaced over one another by a vector which is not an integer linear combination of $\widetilde{\mathbf{b}}_{j}^{\prime}$ s. Only one family out of five, namely, with $Q_{\widetilde{\Delta}}=0$, permits integer indexing in the binary $\widetilde{\mathbf{b}}_{j}$ - basis; we will call the peaks in this family the "main" peaks, the others are "superlattice" peaks (we refer to the 5D superlattice).

The diffraction patterns for our two models (Fig. 12) show that the main peaks dominate the whole diffraction pattern: in this example, the superlattice peak which has the strongest intensity, has only $3 \%$ of the intensity of the strongest main peak. A general theoretical justification of the $\left(Q_{\widetilde{\Delta}}=0\right)$ family domination can be obtained from a general formula for diffraction amplitudes $[5,6]$

$$
A\left(\mathbf{Q}^{\|}\right)=\sum_{\varepsilon=0}^{2} \exp \left(-i \mathbf{Q}^{\|} \cdot \varepsilon \widetilde{\mathbf{b}}_{1}^{\|}\right) \sum_{n_{\Delta}=-2}^{2} \exp \left(-i Q_{\widetilde{\Delta}^{n}} n_{\Delta} / 5\right) \int \exp \left(-i \mathbf{Q}^{\perp} \cdot \mathbf{x}^{\perp}\right) d^{2} \mathbf{x}^{\perp}
$$

where each integral is restricted to one of the fifteen motif 2D-layers. For the peaks of $Q_{\widetilde{\Delta}}=0$, all five layers of each motif add simply with zero relative phases, whereas for other peaks they interfere, which usually decreases the resulting intensity.

On the other hand, the close resemblance of the "main" peaks of the decorated model and all peaks of the undecorated model can be qualitatively attributed merely to the fact that 


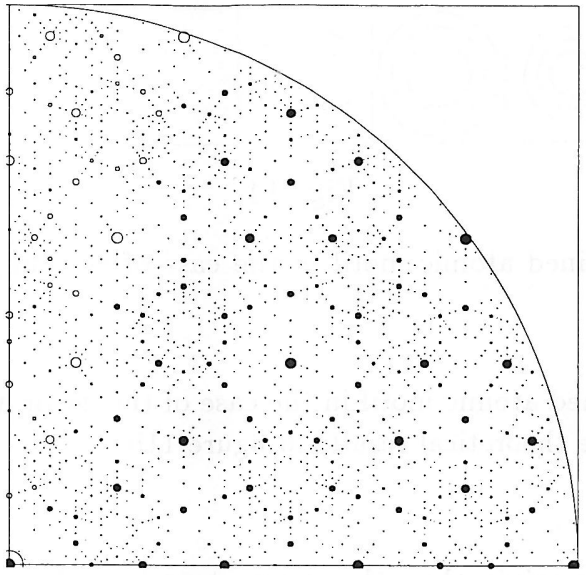

a)

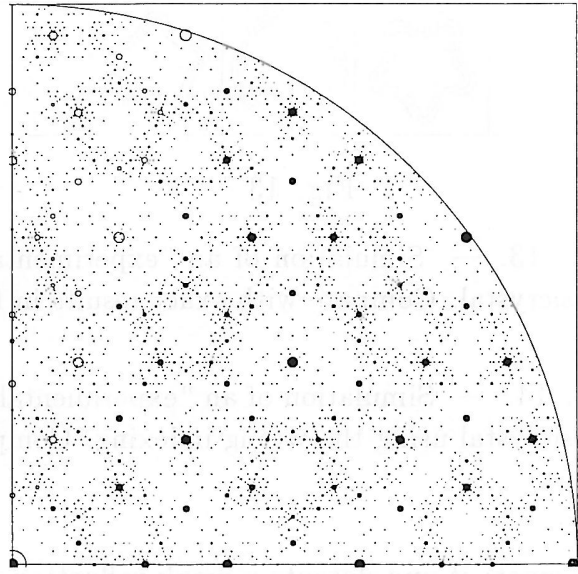

b)

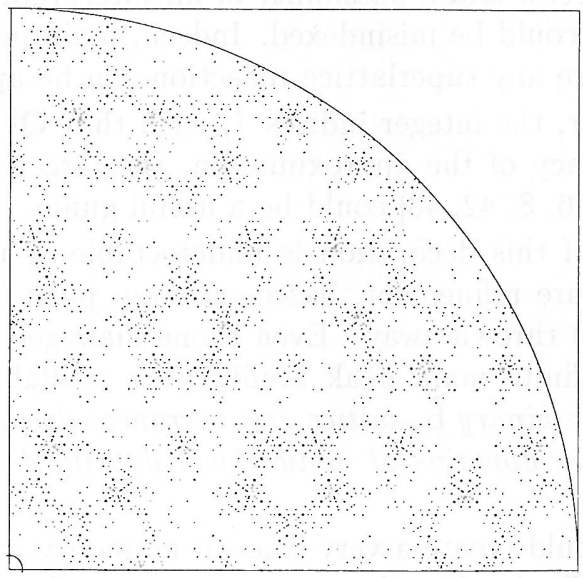

c)

Fig. 12. - Calculated diffraction patterns of simple (a) and decorated (b) quasicrystals (The same unit mass has been put at each vertex of the tiling). Superlattice peaks are shown in (c). The disk areas are proportional to the intensities.

both structures are binary tilings, with the same interatomic distances and elementary tiles. The only difference between the two is in the way the tiles are assembled together. Strong diffraction peaks reflect mainly the crude, short-range features of the structure, the peculiarities in the longer range order manifest themselves only in diffractions with small q-vectors and in satellites. Thus, the common elementary cells of the two models explain the close resemblance of their diffraction patterns.

In principle there is no problem to detect a decorated lattice: there are diffraction spots which cannot be expressed through the binary $\widetilde{\mathbf{b}}_{j}$-basis. However, if one has in hand only a relatively small number of peaks, a mistake is more likely. At worst, the experimental intensity cut-off could even be above the level of the strongest superlattice peak. If so, the mistake would be almost certainly made. But even if some of the superlattice peaks are detectable, they should be recognized as superlattice peaks. In normal crystallography this problem does 


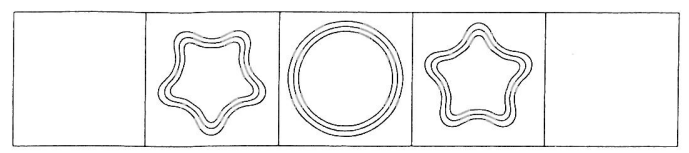

Fig. 13

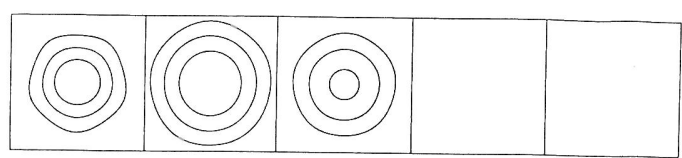

Fig. 14

Fig. 13. - Simulation of an "experimentally" determined atomic motif in the case of the simple quasicrystal. Compare with exact results in figure 10.

Fig. 14. - Simulation of an "experimentally" determined atomic motif in the case of the decorated quasicrystal using the wrong indexing. Compare with the theoretical results in figure $11 \mathrm{~b}$.

not exist: if one detects a weak reflection closer to the origin than the shortest main reciprocal lattice vector, it is a clear indication of a superlattice formed. In quasicrystals there is no shortest reciprocal lattice vector and a possibility of misinterpretation remains: a superlattice peak, even if it is detected, could be misindexed. Indeed, main peaks fill the reciprocal space everywhere densely, therefore any superlattice reflection can be approximated by main peaks with any accuracy. However, the integer indices $f_{j}$ such that $\mathbf{Q}^{\|} \simeq \sum_{j} f_{j} \widetilde{\mathbf{b}}_{j}^{\|}$must be rather large: the better the accuracy of the approximation, the larger the $f_{j}$ 's. The conventional intensity versus $Q^{\perp}$ plot $[5,6,8,42,43]$ could be a useful guide.

However, what happens if this decoration is undetectable or not detected? What would be the outcome of a structure refinement, based on main peak $\left(Q_{\widetilde{\Delta}}=0\right.$ family) only, with superlattice peaks $\left(Q_{\widetilde{\Delta}} \neq 0\right)$ thrown away? Even if one managed to determine all the phases correctly and the whole infinite main peak family were available, an attempt to solve the decorated structure using the binary $\widetilde{\mathbf{b}}_{j}$-lattice (i.e. erroneously presuming only one motif per cell) would result, at best, in domains of nonuniform density deduced from the motifs stuck together, as in figure $11 \mathrm{~b}$.

Truncation effects [16] would create a very smooth boundary and would aggravate such an attempt (Figs. 14 and 15). To simulate the experiment we performed Fourier transform of the truncated $Q_{\widetilde{\Delta}}=0$ subseries $\left(q_{\max }=2 \pi \times 8.5 / b\right.$ where $b$ is the length of the tile edges) and a low intensity cut-off $\left(I_{\min }=0.001 \times I(0)\right)$. The resulting motifs look rather spherical and smooth. It is virtually impossible to recognize the patterns of figure $11 \mathrm{~b}$. Recognition could be possible, in principle, if one used lower intensity cut-off, but with a moderate number of peaks in hand it is more likely to miss such a sophisticated geometry as in figure 11b, substituting it with a simple decagon or the like, as is usually done in real experiments. The problem does not arise in the undecorated structure: figure 13 shows analogous simulated "experimentally" motifs found for undecorated binary tiling; even with a moderate amount of harmonics the stars are quite distinguishable.

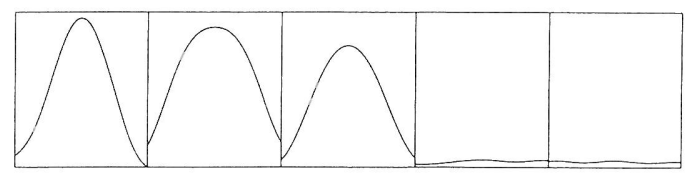

Fig. 15. - Density profile along a five-fold axis of the domains shown in figure 14 (decorated quasicrystal case using the wrong indexing and including truncation effects). 
When this paper was in preparation, we became aware of a recent experiment of Edagawa et al. [22], who reported an observation of the superlattice peaks in the electron diffraction pattern of decagonal AlNiCo. The peaks are indiscernible in poor quality samples; only very careful preparation, specific choice of chemical composition and long annealing create very faint superlattice peaks. The relation of the new (shorter) reciprocal space basis and the basis for main reflections is exactly as in our equation (12) and figure 9b. This allows us to speculate that the phenomenon predicted in the present paper might be indeed observed in this experiment [22]. Steurer et al. [44] and Grushko [45] also report some "superstructure" peaks which cannot be indexed with the conventional basis. Should it be true, the mistake, we warn against here, has been made by one of us (as well as by everybody involved in the decagonal business): AlNiCo is believed to be described by a binary tiling with a simple $5 D$ cubic lattice $[14,15,20]$. Whether or not the result of reference [22] is confirmed, its very appearance justifies our concern: special precautions should be made to detect possible decorated lattice.

\section{Conclusion.}

Hyperspace crystallography as developed in $[7,8]$ may work relatively successfully with simple lattices. A decorated lattice can be easily confused with a simple one; the resulting atomic motifs (obtained erroneously if one assumes that there is only one hyper-atom per unit hypercell) are likely to be smeared and rounded to an extent which does not permit correct carving to polygons. The way to avoid this complication is to pay attention to very careful indexing of extremely faint peaks, by employing electron microscopy if needed, which should reveal the presence of superlattice peaks. There is a certain concern that common difficulties in solving various real quasicrystalline structures might be related to inability to recognize decorated lattices.

\section{Acknowledgments.}

We thank K. Edagawa for informing us about his recent experiment and to B. Grushko for useful discussions. One of us (S.B.) is grateful to the Département de Recherche Fondamentale sur la Matière Condensée of CENG/CEA for kind hospitality and to the Natural Science and Engineering Research Council of Canada for financial support.

\section{References}

[1] Shechtman D., Blech I., Gratias D. and Cahn J.W., Phys. Rev. Lett. 53 (1984) 1951-1953.

[2] Tsai A.P., Inoue A. and Masumoto T., Jpn J. Appl. Phys. 26 (1987) L-1505;

Tsai A.P., Inoue A. and Masumoto T., Jpn J. Appl. Phys. 27 (1988) L-1587;

Tsai A.P., Inoue A. and Masumoto T., Trans. JIM 29 (1988) 521;

Tsai A.P., Inoue A., Yokoyama Y. and Masumoto T., Philos. Mag. Lett. 61 (1990) 9;

Tsai A.P., Inoue A., Yokoyama Y. and Masumoto T., Mat. Trans. JIM 31 (1990) 98.

[3] Guryan C.A., Goldman A.I., Stephens P.W., Hiraga K., Tsai A.P., Inoue A. and Masumoto T., Phys. Rev. Lett. 62 (1989) 2409.

[4] Colella R., private communication.

[5] Methods of Structural Analysis of Modulated Structures and Quasicrystals, J.M. Pérez-Mato, F.J. Zúñiga and G. Madariaga Eds. (World Scientific Publishing Co., Singapore, 1991). 
[6] Quasicrystals: The State of the Art, D.P. DiVincenzo and P.J. Steinhardt Eds. (World Sci., Singapore, 1991).

[7] Gratias D., Cahn J.W. and Mozer B., Phys. Rev. B 38 (1988) 1638;

Gratias D., Cahn J.W. and Mozer B., Phys. Rev. B 38 (1988) 1643.

[8] Janot C., Pannetier J., Dubois J.M. and de Boissieu M., Phys. Rev. Lett. 62 (1989) 450;

Janot C., de Boissieu M., Dubois J.M. and Pannetier J., J. Phys.: Cond. Matt. 1 (1989) 1029.

[9] de Boissieu M., Janot C., Dubois J.M., Audier M. and Dubost B., J. Phys.: Cond. Matt. 3 (1991) 1.

[10] van Smaalen S., de Boer J.L. and Shen Y., Phys, Rev. B43 (1991) 929.

[11] Yamamoto A., Phys, Rev. B 45 (1992) 5217.

[12] Cornier-Quiquandon M., Quivy M., Lefevbre S., Elkami E., Heger G., Katz A. and Gratias D., Phys. Rev. B 44 (1991) 2071.

[13] Boudard M., de Boissieu M., Janot C., Heger G., Beeli C., Nissen H.U., Vincent H., Ibberson R., Audier M. and Dubois J.M., J. Phys.: Cond. Matt. 4 (1992) 10149-10168.

[14] Steurer W. and Kuo K.H., Philos. Mag. Lett. 62 (1990) 175; Steurer W. and Kuo K.H., Acta Cryst. B 46 (1990) 703.

[15] Yamamoto A., Kato K., Shibuya T. and Takeuchi S., Phys. Rev. Lett. 65 (1990) 1603.

[16] de Boissieu M., Janot C. and Dubois J.M., Europhys. lett. 7 (1988) 593

[17] Duneau M. and Oguey C., J. Phys. France 50 (1989) 135.

[18] Cornier-Quiquandon M., Gratias D. and Katz A. in [5], pp. 313-332

[19] Qui S.Y. and Jaric M.V., Quasicrystals, T. Fujiwara and T. Ogawa Eds. (Springer-Verlag, Berlin, 1990) p.48.

[20] Burkov S.E., Phys. Rev. Lett. 67 (1991) 614-617;

Burkov S.E., Physics and Chemistry of Finite Systems: From Clusters to Crystals (NATO ASI Series, v.374), P. Jena, S.N. Khanna and B.K. Rao Eds. (Kluwer, Dordrecht, 1992).

[21] Burkov S.E., Phys. Rev. B 47 (1993) 12325-12328.

[22] Edagawa K., Ichihara M., Suzuki K. and Takeuchi S., Philos. Mag. Lett. 66 (1992) 19.

[23] Lançon F., Billard L. and Chaudhari P., Europhys. Lett. 2 (1986) 625-629.

[24] Lançon F. and Billard L., J. Phys. France 49 (1988) 249-256.

[25] Katz A. and Duneau M., J. Phys. France 47 (1986) 181-196.

Duneau M. and Katz A. Phys. Rev. Lett. 54 (1985) 2688

[26] Elser V., Acta. Crystallogr. A42 (1986) 36-43.

[27] Kalugin P. A., Kitaev A. Yu. and Levitov L. S., JETP Lett. 41 (1985) 145-149.

Kalugin P. A., Kitaev A. Yu. and Levitov L. S., J. Phys. France 46 (1985) L601.

[28] Lançon F. and Billard L., Phase Transitions 44 (1993) 37-46.

[29] Godrèche C. and Luck J.M., Phys. Rev. B 45 (1992) 176-185.

[30] Kramer P., Mod. Phys. Lett. B 1 (1987) 7-18.

Kramer P., Mod. Phys. Lett. B 2 (1988) 605-611.

[31] Oguey C., Duneau M. and Katz A., Commun. Math. Phys. 118 (1988) 99.

[32] Janssen T., Acta Crystallogr. A42 (1986) 261-271.

[33] Bak P., Phys. Rev. Lett. 56 (1986) 861-864.

[34] We thank one of the referees who pointed out this remark.

[35] Widom M., Strandburg K. and Swendsen R.H., Phys. Rev. Lett.58 (1987) 706-709. Strandburg K., Tang L.H. and Jaric M.V., Phys. Rev. Lett. 63 (1989) 314-317. Widom M., Deng D.P. and Henley C.L., Phys. Rev. Lett. 63 (1989) 310-313.

[36] Burkov S.E., J. Phys. I France 2 (1992) 695.

[37] Baake M., Kramer P., Schlottmann M. and Zeidler D., Mod. Phys. Lett. B 4 (1990) 249-258; Baake M., Kramer P., Schlottmann M. and Zeidler D., Int. J. Mod. Phys. B 4 (1990) 2217; Klitzing R., Schlottmann M., and Baake M., Int. J. Mod. Phys. B 7 (1993), 1455-1473. 
[38] Gahler F., private communication, (1992)

[39] de Bruijn N.G., Nederl. Acad. Wetensch. Proc. A84 (1981) 38.

[40] Lück R., J. Non-Cryst. Solids 117/118 (1990) 832-835.

[41] Godrèche C. and Lançon F., J. Phys. I France 2 (1992) 207-220

[42] Cahn J.W., Shechtman D. and Gratias D., J. Mater. Res. 1 (1986) 13.

[43] Elser V., Acta Cryst. A 42 (1986) 36.

[44] Steurer W., Haibach T., Zhang B., Kek S. and Lueck R., submitted to Acta Crystallogr. B (1992).

[45] Grushko B., private communication. 